Published in final edited form as:

Ann Epidemiol. 2007 May ; 17(5): 335-341.

\title{
Skin color and mortality risk among men: The Puerto Rico Heart Health Program
}

\author{
Luisa N. Borrell, DDS, PhD ${ }^{1}$, Carlos J. Crespo, DrPH, MS², and Mario R. Garcia-Palmieri, \\ $\mathrm{MD}^{3}$ \\ 1 Mailman School of Public Health, Columbia University \\ 2 School of Community Health, Portland State University \\ 3 School of Medicine, University of Puerto Rico
}

\section{Abstract}

PURPOSE-To examine the association between skin color and all-cause and CVD-related mortality risk before and after adjusting for selected characteristics and risk factors, we used data on 5,304 men with information on skin color at Exam 3 of the PRHHP, a longitudinal study of the incidence of coronary heart disease in Puerto Rican men, were used.

METHODS—Mortality was ascertained using hospital and physician records, postmortem records, death certificates and information from the next of kin.

RESULTS—Dark skinned men exhibited higher age-adjusted mortality rates than light skinned men (10.1 versus 8.8/10,000 population). There was no association between skin color and all-cause and CVD-related mortality. However, the association between skin color and all-cause mortality varied with area of residence ( $p$ for interaction=0.05). Among men living in urban areas, the risk of allcause mortality was $28 \%$ (95\% CI:1.02-1.61) higher among dark skinned men than their light skinned counterparts after adjusting for age, education, BMI, physical activity and diabetes. There was no association between skin color and CVD mortality in urban men. Neither all-cause nor CVD mortality was associated with skin color among rural men.

CONCLUSION-Our results suggest that skin color may be capturing environmental dynamics that may influence mortality risk among Puerto Rican men.

\section{Keywords}

Skin color; mortality; Puerto Rico; area of residence; Hispanics

In the US, Hispanics (629.3 per 100,000) have lower age-adjusted all-cause death rates than non-Hispanics (856.5). ${ }^{1}$ However, when Hispanic data are disaggregated, a different pattern emerges: Puerto Ricans (766.2) exhibit higher age-adjusted all-cause death rates than Mexican Americans (612.8) and Cubans (524.1) but similar to Hispanics from other countries (761.8). Moreover, a similar pattern has been observed for other health outcomes such as self-rated health and activity limitation status with Puerto Ricans exhibiting the worst outcomes. ${ }^{2}$ These results suggest that heterogeneity among Hispanic subgroups is often lost due to data

Corresponding author: Luisa N. Borrell, DDS, PhD, Assistant Professor, Department of Epidemiology, Mailman School of Public Health, Columbia University, 722 West $168^{\text {th }}$ Street, New York, N. Y. 10032, Ph 212-304-6413, Fax 212-544-4221, Email: lnb2@ columbia.edu

Publisher's Disclaimer: This is a PDF file of an unedited manuscript that has been accepted for publication. As a service to our customers we are providing this early version of the manuscript. The manuscript will undergo copyediting, typesetting, and review of the resulting proof before it is published in its final citable form. Please note that during the production process errors may be discovered which could affect the content, and all legal disclaimers that apply to the journal pertain. 
${ }_{4}^{\text {aggregation }}{ }^{3}$ This aggregation also masks the existing racial heterogeneity among Hispanics.

Although few studies have examined the effect of skin color on health among Hispanics, ${ }^{5-7}$ these studies suggest health differences according to skin color. For example, studies on skin color measured using skin color tiles show that dark-skinned Puerto Rican men had a higher prevalence of left ventricular hypertrophy and slightly higher systolic blood pressure than their light skinned counterparts. 5,6 These studies underscored the role of genetic and environmental determinants of differences between light and dark skinned men while recognizing the limitations of the data. Furthermore, Landale and Oropesa found that mothers' skin color was associated with low birthweight in Puerto Rican infants in selected states in the Northeast region of the US but not in Puerto Rico or New York City. ${ }^{7}$ These findings parallel existing evidence among African Americans, with dark skinned African Americans exhibiting worse health outcomes than light skinned African Americans. ${ }^{8-11}$ Specifically, these studies found a positive association between darker skin color and hypertension. Thus, it is possible that skin color affects the opportunities and resources (or lack thereof) for Hispanics, and this in turn, may influence their health status as it does for African Americans. Therefore, the investigation of skin color, as a proxy for racial categorization, among Puerto Ricans could provide insight into how race works to create health disparities in this population. This racial categorization may be associated with socially patterned experiences such as racism and discrimination.

Data from the Puerto Rico Heart Health Program (PRHHP) study affords the opportunity to investigate the association between skin color and mortality, all-cause and cardiovascular disease (CVD) related deaths in Puerto Rican men before and after adjusting for selected covariates. Specifically, we use data on skin color obtained during Exam 3 and on mortality obtained at the 12-Year follow up of the PRHHP study to investigate: 1) whether skin color measured at Exam 3 is associated with all-cause and CVD-related mortality; and 2) whether these relationships differ by area of residence. We hypothesize that all-cause and CVD-related mortality may be higher among dark skinned men as compared to light skinned men. In addition, because of the difference in social structure between rural and urban areas, we expect that this association could vary with area of residence. Specifically, those living in urban areas at baseline may exhibit stronger associations between mortality and skin color than for those living in rural areas.

\section{Methods}

The PRHHP was designed to examine the epidemiology of coronary heart disease (CHD) including morbidity and mortality associated with CHD in urban and rural Puerto Rican men 35 to 79 years of age at baseline. ${ }^{12,13}$ The study recruited men in March 1965 and followed them up to August 1981. Men were recruited from three urban and four rural municipalities in the northeast part of Puerto Rico using enumeration districts obtained from the US Census.

13 The study's response rate was $80 \%$ of the enumerated individuals at baseline constituting a sample of 9,824 men, including 6,843 from urban municipalities and 2,981 from rural areas. This analysis was limited to participants at Exam 3 (1971-1975) without CHD for whom information on skin color was obtained during the examination. This criteria yielded a sample size of 5,304 men between the ages of 40 to 84 years at Exam 3, with the majority residing in urban areas $(n=3,671)$.

All men completed an extensive interview and a comprehensive physical examination, electrocardiogram, and laboratory exams at baseline. Information on demographic characteristics, medical history and health-related behaviors was collected through a personal interview. 
Skin color was assessed by matching the skin color of the upper inner arm to a set of photographic color tiles, the von Luschan color tiles. ${ }^{5,6}$ These color tiles were used by anthropologists at the time of the study and consisted of 30 colored tiles representing shades of skin color from very light to very dark. The color tiles were matched to each participant's skin color. Recent research found a correlation of 0.95 between measures using electron energy loss (or reflectance spectrophotometry) and the von Luschan's scale adding validity to the use of the latter scale. ${ }^{14}$ To minimize measurement error, all skin color measurements were made by the same examiner. The selection of the upper inner arm avoided any error that may be caused by exposure of the skin to the tropical sun in Puerto Rico. According to previous studies, 5,6 the data on skin color were dichotomized with values 23 to 30 in the scale classified as dark skin and light skin otherwise.

Mortality was ascertained in study participants for whom complete information on skin color was available at the time of the third examination and followed for $6 \frac{1}{4}$ years after that examination. Cause of death was determined using hospital and physician records, postmortem records, death certificates and information from the next of kin. Vital status at year 12 was completed on all but 9 subjects from the initial exam. At the completion of the study (August 1981), there were 552 deaths in participants who had data on skin color and did not have CHD at Exam 3, with 471 deaths occurring in light skinned men and 81 deaths in dark skinned men. For these analyses, deaths were classified according to underlying cause of death as all-cause and CVD-related (cerebrovascular accident, cerebrovascular disease, coronary heart disease and sudden death).

Consistent with previous studies using these data, ${ }^{15,16}$ age, marital status, education, area of residence, smoking, BMI, physical activity, alcohol consumption, cholesterol levels, hypertension and diabetes status were included as covariates. Marital status (single, married, widowed, and divorced or separated), education (less than $8^{\text {th }}$ grade; attended or completed high school; attended or completed college), area of residence (urban/rural), smoking (current, former and never) and alcohol consumption obtained from a 24-hour diet recall interview (none; $1-39 \mathrm{~g}$; and more than $40 \mathrm{~g}$ ) were collected at baseline; while age (continuous), BMI $(\leq 18.5 ; 18.5-24.9 ; 25-29.9 ;$ and $\geq 30)$, physical activity (physical activity index tertiles), cholesterol ( $\leq 200 \mathrm{mg} / \mathrm{dl} ; 200-239 \mathrm{mg} / \mathrm{dl}$; and $\geq 240 \mathrm{mg} / \mathrm{dl}$ ), hypertension (systolic blood pressure $\geq 140 \mathrm{mmHg}$ or diastolic blood pressure $\geq 90 \mathrm{mmHg}$ or currently taking antihypertensive medications) and diabetes (having a non-fasting glucose level $\geq 140 \mathrm{mg} / \mathrm{dl}$ or currently taking medications for diabetes) were collected at baseline and verified at Exam 3. There was little difference in these measures between Exams 1 and 3. For example, BMI at Exam 1 was $25.1 \mathrm{~kg} / \mathrm{m}$ (SE:0.05) and at Exam 3 was $25.5 \mathrm{~kg} / \mathrm{m}$ (SE:0.05). This was also true for diastolic (82.2 [SE:0.16] and 83.8 [SE:0.16] mmHg at Exams 1 and 3, respectively) and systolic blood pressure (132.0 [SE:0.30] and 133.6 [SE:0.32] mmHg at Exams 1 and 3, respectively).

\section{Statistical Analysis}

Descriptive statistics for selected characteristics of the population were calculated by skin color, and further, by all-cause and CVD-related mortality. To determine statistical significance of differences, chi-square tests were used. Poisson regression was used to estimate age-adjusted mortality rates per 10,000 population by skin color and further by area of residence. PROC GENMOD $^{17}$ was used to calculate relative risk (RR) and their $95 \%$ confidence intervals (CI) for all-cause and CVD-related mortality risk in men with dark skin relative to those with light skin, before and after controlling for selected characteristics. In the analysis for CVD, deaths for other causes were treated as censored. The interaction between skin color and area of residence was tested by including the appropriate interaction terms in the models for all-cause and CVD-related mortality. These analyses were repeated for skin color by area of residence 
(urban and rural). Two-sided $p$ values of $<0.05$ were considered statistically significant. Analyses were performed using SAS V9.1.3. ${ }^{18}$

\section{Results}

In general, light skinned Puerto Rican men were more educated, more likely to live in rural areas, have higher cholesterol levels, and a lower prevalence of hypertension than their dark skinned peers (all $p$-values $<0.05$; Table 1 ). There was no association of marital status and alcohol consumption with skin color (data not shown).

Table 2 shows selected characteristics for those who died and those who did not die according to skin color among Puerto Rican men. There were a total of 552 deaths, with 471 among light skinned men and 81 among dark skinned men. When compared to survivors, those who died were more likely to be older, underweight, and have hypertension and diabetes regardless of skin color. In addition, light skinned men who died were more likely to be less educated, while dark skinned men were more likely to live in urban areas and never smoke. With regard to CVD, light and dark skinned men who died were older, more likely to have hypertension and diabetes than those who did not die. Light skinned men who died from CVD had higher income, were more likely to be underweight and had higher cholesterol levels; while dark skinned men were more likely to be never smokers as compared to those who did not die.

Dark skinned men exhibited higher age-adjusted mortality rates (10.1 per 10,000 population) than light skinned men ( 8.8 per 10,000 population, $p=0.23$; Figure 1 ). This pattern persists for men residing in urban areas ( 7.5 for dark skin per 10,000 population versus 6.2 for light skin, $p=0.09)$. However, the opposite was true for men living in rural areas: Light skinned men had at least twice the risk of dying compared with their dark skinned counterparts (14.3 versus 7.0 per 10,000 population, $p=0.10$ ).

Table 3 shows the relative risk and their 95\% CI for the association between skin color and mortality, all-cause and CVD-related mortality, before and after adjusting for selected covariates.

There was no association between skin color and all-cause and CVD-related mortality risk among Puerto Rican men. However, the association between skin color and all-cause mortality varied with area of residence ( $p$ for interaction $=0.05$ ). Among men living in urban areas, the risk of all-cause mortality was $32 \%$ higher among men with dark skin compared to their light skin counterparts. This association remains nearly unchanged (RR:1.28; 95\%CI: 1.02-1.61) after adjusting for age, education, BMI, physical activity, diabetes and area of residence. There was no association between skin color and CVD-related mortality in Puerto Rican men living in urban areas. Similarly, neither all-cause nor CVD-related mortality was associated with skin color among men living in rural areas.

Because of the observed urban-rural difference in mortality among light skinned men (6.2 per 10,000 in urban versus 14.3 in rural), we repeated the analyses for light skinned and dark skinned men separately to evaluate the effect of area of residence on all-cause mortality risk (data not shown in the tables). After adjustment for age, education, BMI, physical activity, hypertension and diabetes, there was no association between area of residence and all-cause mortality among light skined men. However, although the number of events was small for dark skinned men in both urban and rural areas, dark skinned men living in urban areas had a mortality risk 3.11 times (95\% CI: 1.31-7.39) higher than dark skinned men living in rural areas. This finding confirms our skin color/area of residence interaction and suggests that skin color dynamics between the urban and rural areas must be different with urban settings possibly affording a higher risk for dark skin men. 


\section{Discussion}

Our results show no association between skin color and all-cause or CVD-related mortality among Puerto Rican men. However, the association between skin color and all-cause mortality varies with area of residence: Dark skinned Puerto Rican men living in urban areas at baseline have a higher risk of dying from all-cause mortality than their light skinned counterparts. This association was not observed between skin color and CVD-related mortality in men living in urban areas. There was no association between skin color and all-cause or CVD-related mortality for Puerto Rican men living in rural areas.

Results from studies examining the relationship between skin color and health outcomes among African Americans have been inconsistent. ${ }^{8,}{ }^{19-21}$ For example, early studies found an association between skin color and hypertension, with dark skin being associated with hypertension. ${ }^{8}$ The study proposed a biological mechanism based on the presence of tyrosine and dopamine in both melanin and norpeinephrine-epinephrine. ${ }^{8}$ Later studies found no association between skin color and blood pressure among African Americans. These studies suggest the possibility of confounding between skin color and socioeconomic status (SES). 19-21 Specifically, dark skinned African Americans tend to be more likely to be less educated and to have a lower income than their light skinned counterparts. This study also shows that dark skinned Puerto Rican men were less educated than their lighter skinned counterparts. Previous studies using the PRHHP data also have found an association between skin color and the prevalence of left ventricular hypertrophy and systolic blood pressure with darker skinned Puerto Rican men exhibiting worse outcomes than their light skinned counterparts. 5,6

The few studies that focused on the association between skin color and mortality have yielded mixed results. ${ }^{22,} 23$ For example, Keil et al, ${ }^{22}$ using data from the Charleston Heart Study (CHS) in South Carolina, found an association between skin color and all-cause mortality among black men and women with light skinned men and women exhibiting a small increase in risk of dying when compared to their dark skinned counterparts. Using the same CHS data, Knapp et al ${ }^{23}$ examined the association of skin color and cancer mortality. The authors restricted the sample to men only and included a high SES black men supplemental sample. The study found that light skin was protective against cancer mortality only among the high SES subsample. This finding suggested that skin color may be a multidimensional marker that may capture biological and social phenomenon to affect health and disease processes.

Our study did not find an association between skin color and all-cause or CVD-related mortality among Puerto Rican men. However, the association between skin color and all-cause mortality varied with area of residence: Dark skinned Puerto Rican men living in urban areas had a higher risk of dying of all-cause mortality compared to their light skinned counterparts. Although there was no association for men living in rural areas, dark skin appears to be protective in men living in rural areas. Moreover, dark skinned men living in urban areas had a higher all-cause mortality risk than their counterparts living in rural areas. These findings did not support skin color as a genetic marker among Puerto Rican men and the interaction between skin color and area of residence could reflect the social dynamic of skin color among Puerto Ricans. Although Puerto Rico, at the time of the study, retained many of its traditional values, culture and lifestyle, the difference between urban and rural areas in terms of social and economic structure was evident. For example, the urban areas exhibited an industrial growth, a more distinct difference in wealth and poverty and a less cohesive social structure than the rural areas. ${ }^{12}$ Consistent with these changes, it is possible that differential treatment by skin color may have taken place in Puerto Rico as it was happening in the US. Specifically, evidence suggests that skin color among Puerto Rican (dark or non-white versus light or white classification) in New York City $4,24-26$ may have been influencing their life chances in terms of education, income and occupation since the mid 1970s. Similarly, evidence suggests that skin color has been 
considered a marker of discrimination among African Americans with darker skinned individuals reporting higher levels of discrimination than their lighter skinned peers. ${ }^{27,} 28$ Therefore, it is possible that skin color may have acted as a marker for discrimination among Puerto Ricans.

We did not find an association between skin color and CVD-related mortality in Puerto Rican men. However, although previous studies have not examined the association between skin color and CVD-related mortality, previous studies have examined the incidence and mortality of CHD. ${ }^{12,}{ }^{29}$ Specifically, Gordon et al ${ }^{29}$ found a lower incidence of CHD in Puerto Rican men as compared to US whites in the Framingham study and Hawaiians in the Honolulu study. Moreover, Garcia-Palmieri et al ${ }^{12}$ using the PRHHP data did not find an urban-rural difference in CHD mortality over a 12-year period. While our null findings may be due to the small number of deaths related to CVD, they are consistent with prior work. ${ }^{12,} 29$

Among the strengths of our study are the longitudinal design; population-based nature and homogeneity of the sample in terms of characteristics and geographic location; and the availability of information on underlying causes of death, prevalent disease at baseline, and cardiovascular risk factors. Important limitations are the small sample size for the rural area and the small number of events. However, our null findings are consistent with a previous study using these data to examine association between area of residence and mortality. ${ }^{12}$ In addition, there was very little movement from rural to urban areas and vice versa. For example, between Exams 1 and 2 only $1.3 \%$ of the PRHHP men changed their area of residence. This proportion was $1.7 \%$ between Exams 1 and 4 . Therefore, it is unlikely that our findings are solely the result of the small sample size and number of events. Nonetheless, lack of power may have hindered our ability to detect an association, specifically for CVD related mortality. Another limitation was the exclusion of 4,520 men due to loss to follow up $(n=1,506)$ at Exam 3, presence of CHD at Exam $3(n=421)$ or lack of data on skin color $(n=2,410)$. This exclusion could have biased our results. We compared the distribution of risk factors between participants with and without skin color information and the only significant differences were for BMI and physical activity. Men without skin color data were more likely to be underweight and less physically active. However, there was no difference in all-cause mortality risk between men with and without skin color information. Therefore, it is very unlikely that their exclusion could have affected the results. Although the measurements of skin color were taken six years before the mortality assessments, skin color is likely to remain relatively stable over time. ${ }^{30-34}$ However, if skin color changed substantially during this time, and thus, led to more or less discrimination, our results regarding associations of skin color with mortality could have been either under- or over-estimated. Moreover, it is possible that the examiner was not blinded to the social status of the participants and was more likely to record high social status participants as light skinned. However, this is unlikely, because social status in the PRHHP was based on self-reported education, occupation and income at baseline, and not on data originated from the examiner at Exam 3. Moreover, if this bias occurred, it would have underestimated our results. Similarly, it is possible that social status was associated with sun exposure with high social status men being less likely to be exposed to sun, and thus, be more likely to appear lighter than they would if exposed to the sun. Again, this may have underestimated our results.

Our study found that skin color was associated with an increase risk of all-cause mortality among urban Puerto Rican men only. Dark skinned men exhibited a higher risk of dying than their light skinned counterparts. Our results suggest that skin color may have captured environmental dynamics that may have influenced mortality risk in this homogenous population. Given the US influence in Puerto Rico over the past 30 years (i.e., racial dynamics), research replicating these results in Puerto Rico is imperative. 


\section{Acknowledgements}

The Puerto Rico Heart Health Program (PRHHP) was conducted and supported by the NHLBI in collaboration with the PRHHP Registry Investigators. Additionally, this work was supported by the National Institute Dental and Craniofacial Research Grant K22DE15317 (LNB), the Robert Wood Johnson Health and Society Scholars Program (LNB), the Department of Defense DAMD17-02-1-0252 (CJC), and the National Institutes of Health 1P20CA96256-01A1 (CJC) and 1R03 CA103475-01 (CJC). The authors would like to thank Dr. Paul Sorlie for his helpful comments on an early draft of this manuscript and Mr. Sean Coady for his help getting the data. This Manuscript was prepared using a limited access dataset obtained by the NHLBI and does not necessarily reflect the opinions or views of the PRHHP Registry or the NHLBI.

\section{References}

1. Kochaneck, KD.; Murphy, SL.; Anderson, RN. Deaths: Final Data for 2002. Hyattsville, MD: National center for Health Statistics; 2004.

2. Hajat A, Lucas JB, Kington R. Health outcomes among Hispanic subgroups: data from the National Health Interview Survey, 1992-95. Adv Data 2000;(310):1-14. [PubMed: 10977762]

3. Vega WA, Amaro H. Latino outlook: good health, uncertain prognosis. Annual Review of Public Health 1994;15:39-67.[Review] [126 refs]

4. Rodriguez, CE. Changing Race: Latinos, the Census, and the history of ethnicity in the United States. New York, NY: New York University Press; 2000.

5. Costas R Jr, Garcia-Palmieri MR, Sorlie P, Hertzmark E. Coronary heart disease risk factors in men with light and dark skin in Puerto Rico. Am J Public Health 1981;71(6):614-9. [PubMed: 7235099]

6. Sorlie PD, Garcia-Palmieri MR, Costas R Jr. Left ventricular hypertrophy among dark- and lightskinned Puerto Rican men: the Puerto Rico Heart Health Program. Am Heart J 1988;116(3):777-83. [PubMed: 2970772]

7. Landale NS, Oropesa RS. What does skin color have to do with infant health? An analysis of low birth weight among mainland and island Puerto Ricans. Soc Sci Med 2005;61(2):379-91. [PubMed: 15893053]

8. Boyle E Jr. Biological pattern in hypertension by race, sex, body weight, and skin color. Jama 1970;213 (10):1637-43. [PubMed: 5468705]

9. Harburg E, Gleibermann L, Roeper P, Schork MA, Schull WJ. Skin color, ethnicity, and blood pressure I: Detroit blacks. American Journal of Public Health 1978;68(12):1177-83. [PubMed: 736181]

10. Gillum RF. Pathophysiology of hypertension in blacks and whites. A review of the basis of racial blood pressure differences. Hypertension 1979;1(5):468-75. [PubMed: 541040]

11. Gleiberman L, Harburg E, Frone MR, Russell M, Cooper ML. Skin colour, measures of socioeconomic status, and blood pressure among blacks in Erie County, NY. Annals of Human Biology 1995;22(1):69-73. [PubMed: 7762977]

12. Garcia-Palmieri MR, Sorlie PD, Havlik RJ, Costas R Jr, Cruz-Vidal M. Urban-rural differences in 12 year coronary heart disease mortality: the Puerto Rico Heart Health Program. J Clin Epidemiol 1988;41(3):285-92. [PubMed: 3339382]

13. Garcia-Palmieri MR, Feliberti M, Costas R Jr, et al. An epidemiological study on coronary heart disease in Puerto Rico: The Puerto Rico Heart Health Program. Bol Asoc Med P R 1969;61(6):1749. [PubMed: 5257818]

14. Henneberg M, Brace CL. Human skin color as a measure of time in situ. American Journal of Physical Anthropology 2000:177. [PubMed: 10640945]

15. Garcia-Palmieri MR, Crespo CJ, Mc Gee D, Sempos C, Smit E, Sorlie PD. Wide pulse pressure is an independent predictor of cardiovascular mortality in Puerto Rican men. Nutr Metab Cardiovasc Dis 2005;15(1):71-8. [PubMed: 15871854]

16. Crespo CJ, Palmieri MR, Perdomo RP, et al. The relationship of physical activity and body weight with all-cause mortality: results from the Puerto Rico Heart Health Program. Ann Epidemiol 2002;12 (8):543-52. [PubMed: 12495827]

17. Spiegelman D, Hertzmark E. Easy SAS Calculations for Risk or Prevalence Ratios and Differences. Am J Epidemiol 2005;162(3):199-200. [PubMed: 15987728]

18. SAS Institute Inc. SAS/STAT 9.1 User's Guide. Cary, NC: SAS Institute Inc; 2004. 
19. Keil JE, Tyroler HA, Sandifer SH, Boyle E Jr. Hypertension: effects of social class and racial admixture: the results of a cohort study in the black population of Charleston, South Carolina. American Journal of Public Health 1977;67(7):634-9. [PubMed: 879392]

20. Keil JE, Sandifer SH, Loadholt CB, Boyle E Jr. Skin color and education effects on blood pressure. American Journal of Public Health 1981;71(5):532-4. [PubMed: 7212145]

21. Klag MJ, Whelton PK, Coresh J, Grim CE, Kuller LH. The association of skin color with blood pressure in US blacks with low socioeconomic status. JAMA 1991;265(5):599-602. [PubMed: 1987409]

22. Keil JE, Sutherland SE, Knapp RG, Tyroler HA, Pollitzer WS. Skin color and mortality. Am J Epidemiol 1992;136(11):1295-302. [PubMed: 1488957]

23. Knapp RG, Keil JE, Sutherland SE, Rust PF, Hames C, Tyroler HA. Skin color and cancer mortality among black men in the Charleston Heart Study. Clin Genet 1995;47(4):200-6. [PubMed: 7628122]

24. Rodriguez CE. Puerto Ricans: Between Black and White. New York Affairs 1974;1(4):92-101.

25. Rodriguez, CE. The effect of race on Puerto Rican wages. In: Melendez, E., editor. Hispanics in the Labor Force. New York, NY: Plenum Press; 1991. p. 77-98.

26. Rodriguez CE. Race, Culture, and Latino Otherness in the 1980 Census. Social Science Quarterly 1992;73(4):930-7.

27. Klonoff EA, Landrine H. Is skin color a marker for racial discrimination? Explaining the skin colorhypertension relationship. J Behav Med 2000;23(4):329-38. [PubMed: 10984862]

28. Keith VM, Herring C. Skin Tone and Stratification in the Black-Community. American Journal of Sociology 1991;97(3):760-78.

29. Gordon T, Garcia-Palmieri MR, Kagan A, Kannel WB, Schiffman J. Differences in coronary heart disease in Framingham, Honolulu and Puerto Rico. J Chronic Dis 1974;27(7-8):329-44. [PubMed: 4436425]

30. Clark P, Stark AE, Walsh RJ, Jardine R, Martin NG. A twin study of skin reflectance. Ann Hum Biol 1981;8(6):529-41. [PubMed: 7199888]

31. Dwyer T, Muller HK, Blizzard L, Ashbolt R, Phillips G. The use of spectrophotometry to estimate melanin density in Caucasians. Cancer Epidemiol Biomarkers Prev 1998;7(3):203-6. [PubMed: 9521433]

32. Jablonski NG, Chaplin G. The evolution of human skin coloration. J Hum Evol 2000;39(1):57-106. [PubMed: 10896812]

33. Shriver MD, Parra EJ. Comparison of narrow-band reflectance spectroscopy and tristimulus colorimetry for measurements of skin and hair color in persons of different biological ancestry. Am J Phys Anthropol 2000;112(1):17-27. [PubMed: 10766940]

34. van der Mei IA, Blizzard L, Stankovich J, Ponsonby AL, Dwyer T. Misclassification due to body hair and seasonal variation on melanin density estimates for skin type using spectrophotometry. J Photochem Photobiol B 2002;68(1):45-52. [PubMed: 12208036]

\section{Selected abbreviations and Acronyms}

PRHHP

Puerto Rico Heart Health program

CVD

Cardiovascular disease

CHD

Coronary heart disease

BMI

Body mass index

$\mathbf{R R}$

Relative risk 


\section{CI}

Confidence intervals 


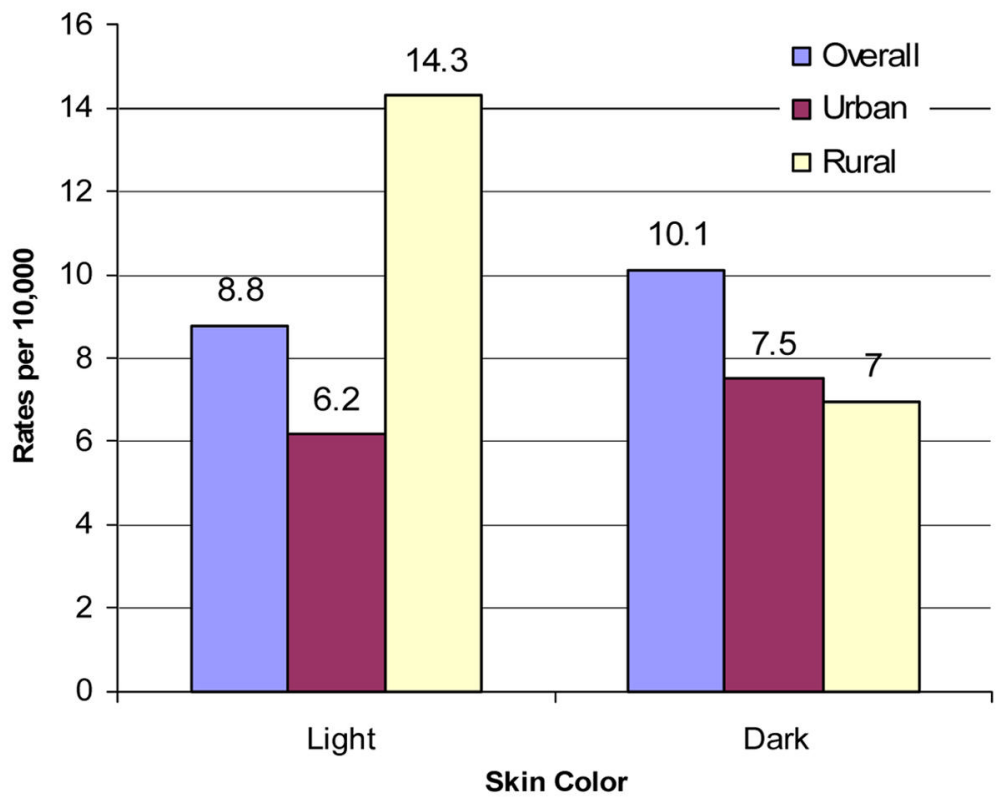

Figure 1.

Age-adjusted death rates according to skin color and area of residence: The PRHHP Study 


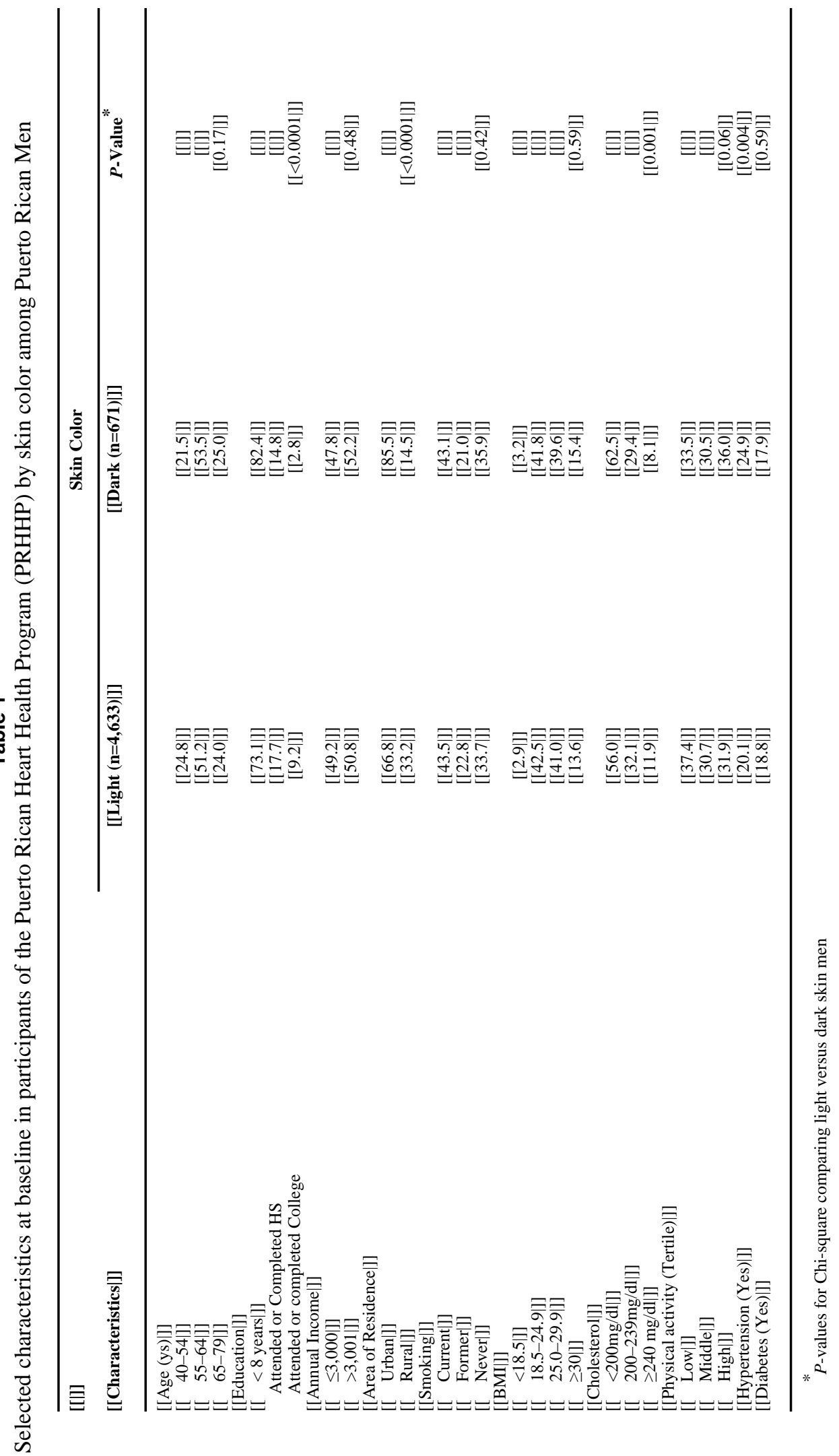




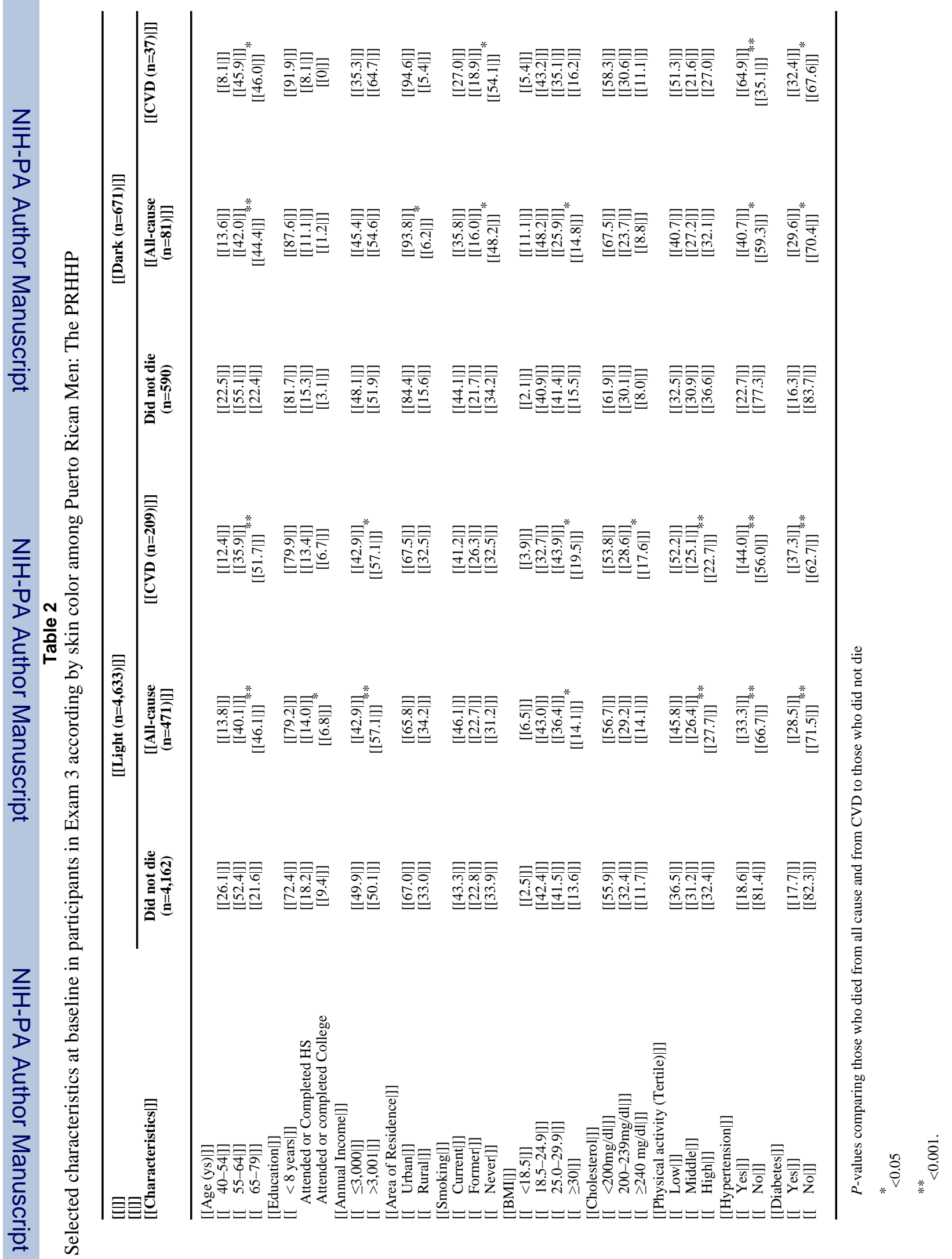




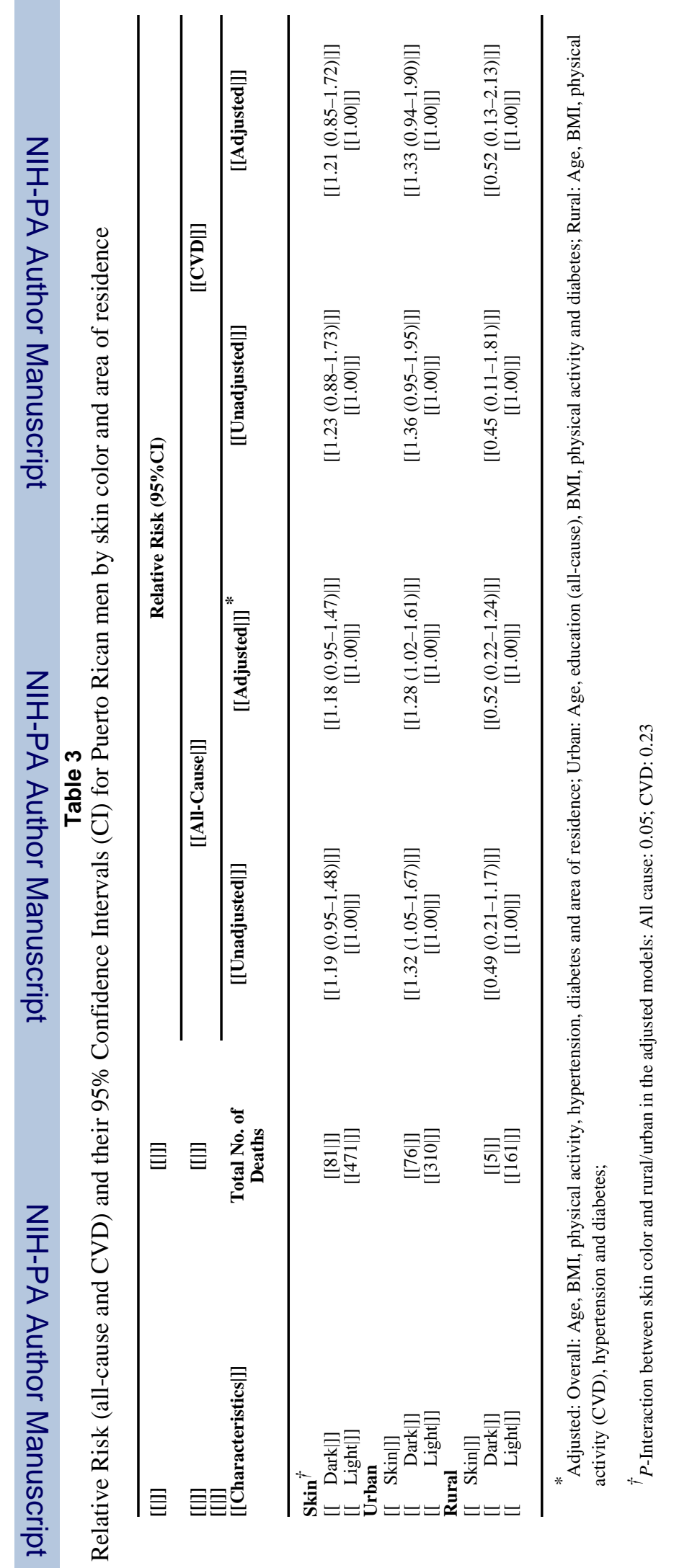

